\title{
Molecular profiling of pediatric meningiomas shows tumor characteristics distinct from adult meningiomas
}

\author{
Elmar Kirches ${ }^{1} \cdot$ Felix Sahm ${ }^{3} \cdot$ Andrey Korshunov $^{3} \cdot$ Christina Bluecher $^{1} \cdot$ Natalie Waldt $^{1} \cdot$ Siegfried Kropf $^{2}$. \\ Daniel Schrimpf ${ }^{3} \cdot$ Philipp Sievers $^{3} \cdot$ Damian Stichel $^{3}$. Ulrich Schüller ${ }^{4,5}$. Jens Schittenhelm ${ }^{6}$. \\ Markus J. Riemenschneider ${ }^{7}$ Matthias A. Karajannis ${ }^{15,18} \cdot$ Arie Perry $^{16} \cdot$ Torsten Pietsch $^{8} \cdot$ Svenja Boekhoff $^{9}$. \\ David Capper ${ }^{10,11} \cdot$ Katja Beck $^{12}$. Nagarajan Paramasivam ${ }^{12}$ - Matthias Schlesner ${ }^{12,20}$. Priscilla K. Brastianos ${ }^{17}$. \\ Hermann L. Müller ${ }^{9} \cdot$ Stefan M. Pfister ${ }^{13,14}$. Christian Mawrin ${ }^{1,19}$
}

Received: 29 July 2020 / Revised: 10 July 2021 / Accepted: 18 July 2021 / Published online: 8 September 2021

(c) The Author(s) 2021

\begin{abstract}
In contrast to adults, meningiomas are uncommon tumors in childhood and adolescence. Whether adult and pediatric meningiomas differ on a molecular level is unclear. Here we report detailed genomic analyses of 37 pediatric meningiomas by sequencing and DNA methylation profiling. Histologically, the series was dominated by meningioma subtypes with aggressive behavior, with $70 \%$ of patients suffering from WHO grade II or III meningiomas. The most frequent cytogenetic aberrations were loss of chromosomes 22 (23/37 [62\%]), 1 (9/37 [24\%]), 18 (7/37 [19\%]), and 14 (5/37 [14\%]). Tumors with NF2 alterations exhibited overall increased chromosomal instability. Unsupervised clustering of DNA methylation profiles revealed separation into three groups: designated group 1 composed of clear cell and papillary meningiomas, whereas group 2A comprised predominantly atypical meningiomas and group 2B enriched for rare high-grade subtypes (rhabdoid, chordoid). Meningiomas from NF2 patients clustered exclusively within groups 1 and 2A. When compared with a dataset of 105 adult meningiomas, the pediatric meningiomas largely grouped separately. Targeted panel DNA sequencing of 34 tumors revealed frequent $N F 2$ alterations, while other typical alterations found in adult non-NF2 tumors were absent. These data demonstrate that pediatric meningiomas are characterized by molecular features distinct from adult tumors.
\end{abstract}

Keywords Meningioma $\cdot$ Methylation profile $\cdot$ Targeted sequencing $\cdot$ NF2

\section{Introduction}

Among adult patients, meningiomas represent the most common primary intracranial tumor, accounting for $36.6 \%$ of newly diagnosed primary brain tumors [17]. In contrast, meningiomas are rare among children and young adults, accounting for only $3-5 \%$ of intracranial neoplasms [16, 17].

Prior clinical and genetic studies have suggested that differences exist between pediatric and adult meningiomas in regard to anatomical site and clinical behavior [20, 21, 26]. While the vast majority of adult patients with meningiomas

Elmar Kirches, Felix Sahm, Andrey Korshunov, Stefan M. Pfister and Christian Mawrin contributed equally.

Christian Mawrin

christian.mawrin@med.ovgu.de

Extended author information available on the last page of the article presents with sporadic tumors, pediatric patients are more commonly affected by an underlying tumor predisposition syndrome, mainly NF2. One study showed that pediatric meningiomas harbored $N F 2$ deletions frequently ( $82 \%$ ) along with more aggressive histological features (high mitotic count, brain invasion) [21]. The same study reported much higher frequencies of deletions in DAL-1, 1p, and 14q compared to adult counterparts. Recently it was reported that some pediatric meningiomas may carry YAP1 fusions [35].

Due to the significant risk of underlying NF2, young patients presenting with a solitary meningioma should be referred for human genetic counseling focused on NF2 surveillance including genetic testing [19] Besides sporadic and NF2-related meningiomas, radiation induced meningiomas represent another clinically distinct subset [32].

The knowledge regarding the molecular alterations in adult meningiomas has been increased substantially over the last 5 years. While the fundamental role of biallelic 
NF2 loss in meningiomas from NF2 patients and approximately $50 \%$ of sporadic meningiomas has long been recognized [27, 28], a spectrum of recurrent oncogenic driver mutations including in SMO, KLF4, TRAF7, AKT1, or $P I K 3 C A$ has more recently been identified, and are mutually exclusive with $N F 2$ alterations. Clinically relevant associations between driver mutations, anatomical site and histological subtype have been recognized, and are reviewed in [23]. Some of these mutations such as in the $S M O$ or $A K T 1$ genes are now considered for molecular targeted therapies in case of recurrent or inoperable disease [23]. Similar to meningiomas arising in the setting of germline NF2 mutations, radiation induced meningiomas are characterized by $N F 2$ inactivation [1]. Recently, based on methylation profiling six different subgroups of meningiomas in adults have been introduced, which differ by site, histological subtype, and prognosis [31].

In contrast, studies addressing the molecular alterations in pediatric meningiomas by comprehensive highthroughput analyses are rare. One study failed to detect the commonly observed mutations in $A K T 1, S M O, K L F 4$, and TRAF7 (exon 17) by using conventional Sanger sequencing [3], and further comprehensive studies have not been published. Here we report the results from a comprehensive analysis of 37 pediatric meningiomas, integrating clinicopathological with genomic data derived from DNA methylation profiling and targeted panel sequencing.

\section{Materials and methods}

\section{Tumor material}

We analyzed 41 samples from 37 patients with meningioma (age range: 1-21 years, median age: 10 years). The study was approved by the local institutional review board of the University Hospital Magdeburg (\#19/14). For 32 patients, only formalin-fixed paraffin-embedded (FFPE) samples were available for histological analysis. Tumors were graded according to the current 2016 WHO classification of brain tumors [14]. Ki-67 proliferation index of the tumors was assessed immunohistochemically using standard procedures. Tumor localization was stratified into convexity, skull base, spinal, and intraventricular sites. Clinical information regarding NF2 status was available for 32 patients, seven of which met clinical diagnostic criteria for NF2. Information regarding tumor recurrence was available for 17 patients. In four patients, prior CNS irradiation for tumor therapy (retinoblastoma, medulloblastoma, ependymoma, ALL) suggests that the meningiomas are secondary neoplasms.

\section{Immunohistochemistry [tissue microarray (TMA)]}

From a total of 19 cases, we generated two TMAs as previously described [18]. The following antibodies were subjected to TMA samples: Akt/phospho-Akt, MAPK/phosphoMAPK, mTOR, phospho-p70S6K, 4EBP/phospho-4EBP, PDGF receptor, p38MAPK, TGF $\beta R 2$, E-Cadherin, and MMP9 (all from Cell Signaling, MA).

\section{Sanger sequencing}

DNA extracted from FFPE tissue was processed using standard procedures as previously described [42]. We analyzed all meningiomas for known hotspot mutations in the genes $S M O$ (L412F and W535L) [4], AKT1 (E17K) [42], and KLF4 (K409Q) [41].

\section{$450 \mathrm{~K}$ methylation profiling}

For methylation analysis and copy-number analysis of the samples, Illumina $450 \mathrm{k}$ Human BeadChip (Illumina, San Diego, CA, USA) analyses were performed as previously described [38].

\section{Panel sequencing analysis}

Panel sequencing for 130 genes reported to be mutant in meningioma based on a literature search done in October, 2014 was done by applying a custom hybrid-capture approach (Agilent Technologies, CA, USA) as described previously [29]. The panel was designed to assess the frequency of known mutations in the respective methylation classes and did not aim to detect novel mutational events.

\section{Whole genome sequencing}

DNA libraries were prepared from tumor and matched normal tissue (blood) available for five pediatric meningioma cases (three with recurrent tumor samples available) and sequenced on three lanes each on HiSeq2000 instruments $(2 \times 100 \mathrm{bp})$. DNA libraries were prepared according to the Illumina TruSeq Nano DNA Library protocol using the TruSeq DNA Nano kit (Illumina, Hayward, CA) and sequenced on one lane on HiSeq X $(2 \times 151 \mathrm{bp})$ using the HiSeq X Ten Reagent Kit v2.5 (both Illumina).

\section{Alignment and detection of small variants}

The raw reads were mapped to the human reference genome (build 37, version hs37d5), using BWA mem (version 0.7.15, with parameter $-T 0$ ), sorted using SAMtools (version 
0.1.19), and duplicate reads were marked using Sambamba (version 0.6.5, with parameter $-t 1-10$-hash-tablesize $=2,000,000$-overflow-list-size $=1,000,000$-io-buffersize $=64)$. Using the tumor and corresponding matched normal samples, somatic small variants (SNVs and indels) were called using the in-house pipelines as described earlier [32]. Briefly, somatic SNVs were called using SAMtools mpileup (version 0.1.19, with parameters -REI -q $30-\mathrm{ug}$ ) and bcftools on tumor sample and then queried in the control samples (with parameters -ABRI -Q $0-\mathrm{q} 1$ ). To enable calling of variants with low allele frequency we disabled the Bayesian model in bcftools (by setting $-\mathrm{p} 2$ ). The raw calls were annotated with ANNOVAR and many publicly available tracks such as 1000 Genome variants, single nucleotide polymorphism database (dbSNP), genomic repeat and low complexity regions and locally available controls. Confidence scores for these variants were annotated as described previously [11]. Indels were called using Platypus (version 0.8.1, with parameters -bufferSize $=100,000$ - maxReads $=5,000,000$ ), and were annotated similar to the somatic SNVs. High confidence somatic indels were required to have the genotype 0/0 (homozygous to reference allele) in the control sample and the platypus filter tag 'PASS' or, to enable the detection of somatic variants with low allele frequency, pass custom filters when Platypus reported 'allele bias'.

Somatic small variants misclassified as germline due to contamination of the matched normal samples with tumor DNA/cells were rescued using the in-house tool TiNDA (Tumor in Normal Detection Analysis). VAFs from variants which are classified as 'germline' (i.e. variant reads have been identified in both tumor and the matched normal sample), and which are novel or rare (minor allele frequency (MAF) $<0.001$ in gnomAD (version 2.0.1) and not present in the above local control of 280 WGS samples) were clustered using EM-based unsupervised clustering from Canopy (version 1.2.0) [10]. Clusters in which at least $85 \%$ of the members have a tumor variant allele frequency (VAF) of at least 0.01 and a matched control VAF below 0.45 were considered as misclassified somatic variants. These rescued somatic SNVs and indels were mapped to the mpileup and Platypus raw calls and variants with confidence score $>7$ were merged into the final somatic small variant calls. Variants in the remaining clusters were classified as rare germline and were annotated as rare high confidence germline variants if they had a confidence score $>7$ in the corresponding raw calls.

\section{Structural variant detection}

Genomic structural variants were detected using SOPHIA (version 34.0; https://bitbucket.org/utoprak/sophia) as described earlier [32], using a background population database consisting of 3261 WGS controls across different diseases (published TCGA cohorts and published/unpublished DKFZ cohorts) and sequencing technologies (100 bp read length Illumina HiSeq 2000/2500 and 151 bp read length Illumina HiSeq X) aligned using the same alignment settings and workflow as used in the present study. Gencode V19 was used for the gene annotations.

Copy number states were called and tumor purity and ploidy were estimated using ACEseq (Allele-specific copynumber estimation from sequencing; https://www.biorxiv. org/content/early/2017/10/29/210807) as described previously [32]. In cases where ACEseq provides multiple purity and ploidy solutions, the lowest ploidy solution which allowed to fit the majority of genomic segments to integer copy numbers and which was consistent with the mutant allele frequency distribution of somatic SNVs was manually selected.

\section{Statistical analyses}

Statistical analyses were performed using IBM SPSS Statistics, version 25 (IBM Corporation and its licensors, 1989, 2017) with a nominal error level of $5 \%$. According to the type of variables (categorial, metrical or time-to-event endpoints), comparisons were carried out in chi-square, Mann-Whitney or log rank tests. For pairwise comparisons with three groups, the closed testing procedure was used (global test followed by pairwise comparisons) in categorial and time-to-event data and the Kruskal-Wallis tests including adjusted pairwise tests for metrical data. However, due to the limited number of available cases and the exploratory aim of the analyses, no adjustment for considering multiple endpoints has been made.

For unsupervised hierarchical clustering of 37 histologically defined pediatric meningioma and 105 reference samples, we selected the 10,000 most variably methylated $\mathrm{CpG}$ sites across the dataset according to median absolute deviation. Pairwise similarity of samples was calculated using Euclidean distance. Clusters were then linked according to the Ward's linkage method.

\section{Results}

The histological subtype distribution among the 37 primary pediatric meningiomas which were graded according to the 2016 WHO classification [14] of brain tumors is shown in Fig. 1a-c. While 30\% of tumors were of WHO grade I, the largest group consisted of atypical WHO grade II meningiomas (57\%). Among 5 WHO grade III meningiomas (14\%), three tumors were rhabdoid meningiomas. Thus, pediatric meningiomas are predominantly characterized by a more aggressive histology than WHO grade I, 
a

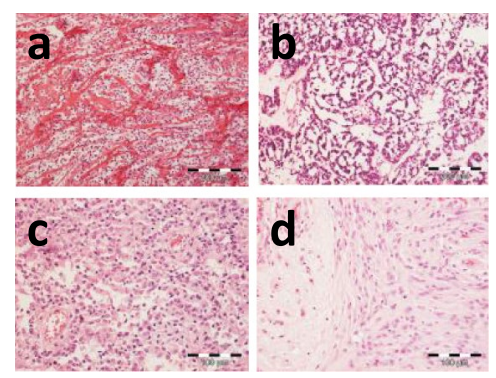

b

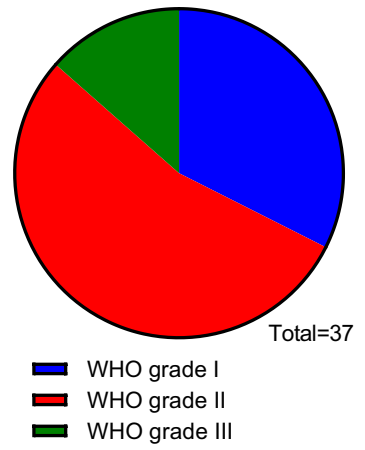

C

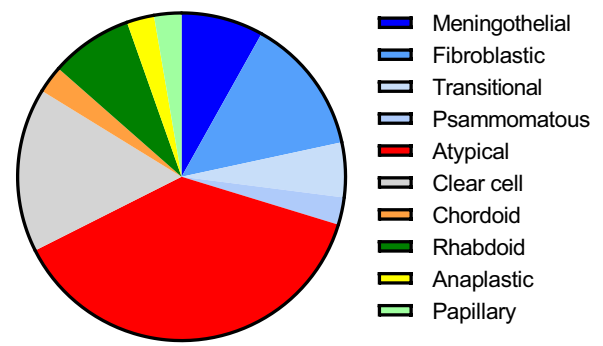

d

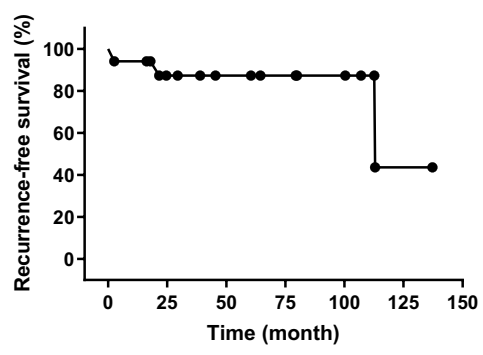

g

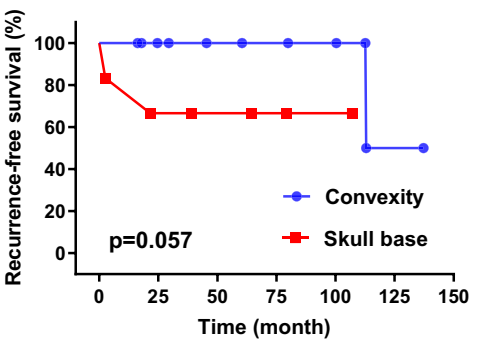

e

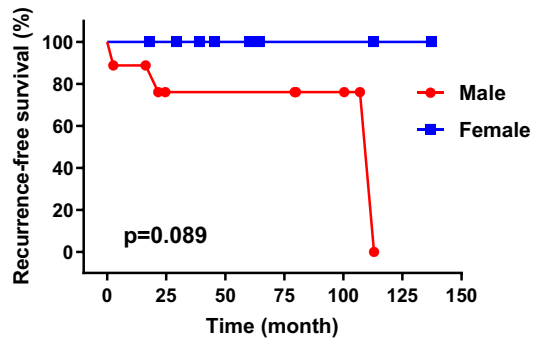

f

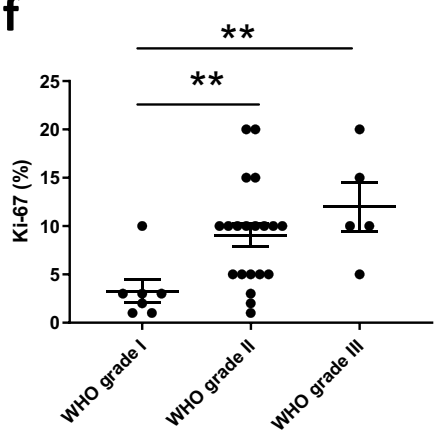

Fig. 1 Pediatric meningiomas are aggressive tumors with uncommon histological features. a Examples of histological subtypes more frequent among pediatric meningiomas than in adult tumors. a Clear cell meningioma WHO grade II. b Chordoid meningioma WHO grade II. c Rhabdoid meningioma WHO grade III. d Atypical meningioma WHO grade II with brain invasion. Distribution by WHO grade (b) and histological variants (c). d, e Kaplan-Meier curves showing clinical characteristics of 17 pediatric meningiomas with follow-up data available. f Proliferation activity as determined by Ki-67 immunostaining within different WHO grades. g Recurrence-free survival according to the tumor localization (skull base or convexity)

shown. As shown in Fig. 1e, male patients had a trend to less favorable outcome than female patients $(p=0.089)$. Proliferation activity as counted by Ki-67 staining was significantly higher in WHO grade II and grade III tumors than in WHO grade I meningiomas (Fig. 1f). Except one patient with spinal meningioma (2.7\%), 36 tumors $(97.3 \%)$ were located intracranially. Most tumors were found at the convexity $(N=18,48.6 \%)$, followed by skull base $(N=15$, $40.5 \%)$ and ventricular system $(N=3,8.1 \%)$. Skull base 
localization was associated with slightly less favorable prognosis compared to convexity localization $(p=0.057$, Fig. 1g).

\section{Somatic and germline NF2 alterations are the dominating molecular event in pediatric meningiomas}

In an initial attempt, we wondered whether recurrent somatic mutations reported to occur in adult meningiomas exclusive of $N F 2$ alterations are present in pediatric meningiomas as well $[5,7,25]$. However, by Sanger sequencing, we did not detect mutations at the established hotspots in $A K T 1, S M O$, KLF4 and TRAF7, suggesting different tumor drivers acting in this age group. This observation prompted us to further analyze the samples by WGS, DNA methylation profiling, and panel sequencing.

First, we subjected all 37 patient samples to methylation profiling and DNA copy-number analysis. The most frequent alteration was loss of chromosome 22 including the $N F 2$ gene $(N=23,62.2 \%)$, followed by loss of chromosomal material on chromosome $1(N=9,24.3 \%)$, chromosome $18(N=7,18.9 \%)$, and chromosome $14(N=5,13.5 \%)$ (Fig. 2a-c). The distribution among the WHO grades, as well as between males and females is shown in Fig. 2d, e.

Because loss of chromosome 22 turned out to be the most frequent molecular event in our series, tumors with (NF2) or without (Non-NF2) alterations were compared (Fig. 3). Comparing the $\mathrm{CNV}$ profile between both groups,
NF2-altered tumors (Fig. 3a) had a higher rate of CNVs than Non-NF2 tumors (Fig. 3b), irrespective of WHO grade (Fig. 3c). The highest frequency of CNVs among NF2altered tumors was found in the WHO grade II group. NF2altered tumors tend to have higher proliferation activity (Fig. 3d). In contrast to chromosomal losses, gain of chromosomal material was seen infrequently. Gain of chromosome $11(p=0.042)$ and chromosome $21(p=0.015)$ were independently associated with higher tumor grading. The presence of tumor necrosis was significantly associated with gain of chromosome $13(p=0.024)$. The histological variant was significantly associated with gain of chromosome $12(p=0.034)$, showing gains in one anaplastic, one atypical, and one chordoid meningioma, respectively. In line with this, gain of chromosome 12 was additionally associated with increased number of mitoses $(p=0.09)$. Gain of chromosome 14 was associated with higher mitotic activity $(p=0.05)$ as well. All tumors showed an unmethylated $M G M T$ promoter.

Whole genome sequencing could be performed for a total of five meningiomas. Three of them showed germline $N F 2$ alterations together with somatic loss of the second allele, while two cases showed somatic NF2 mutations. From three cases (case \#3 \& \#8 with germline NF2 alteration, case \#6 with somatic $N F 2$ alteration), primary and recurrent tumors were available for WGS as well. As shown in supplementary Figure S2, recurrent tumors were similar to the primary tumors with the exception that the second and third recurrence from tumor \#8, as well as recurrence from tumor \#3 had an additional amplification of the LZTRI gene. a

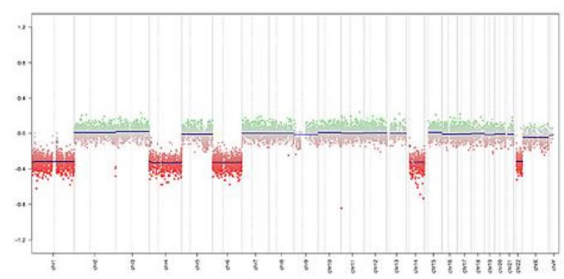

b

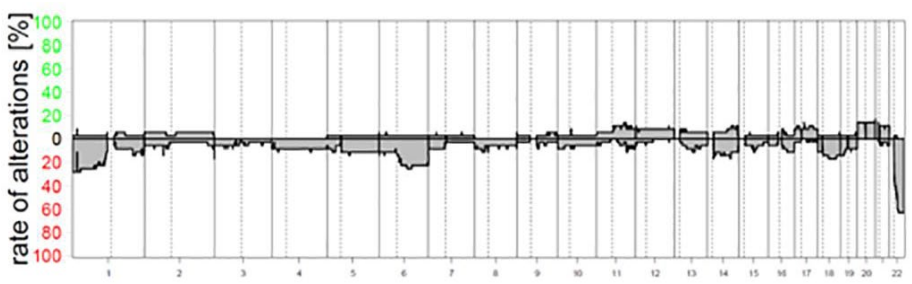

C

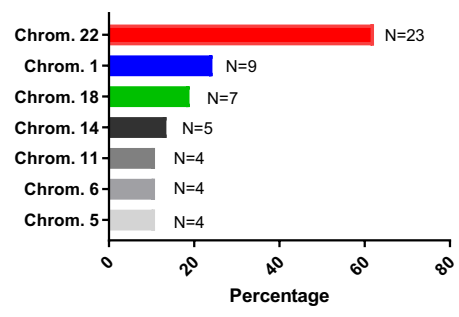

d

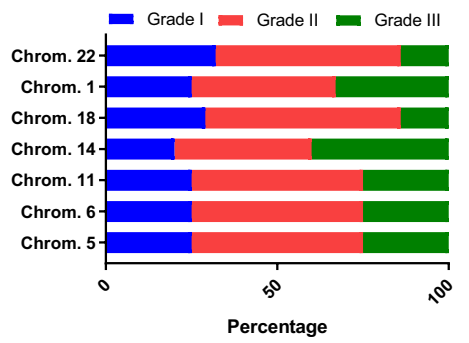

e

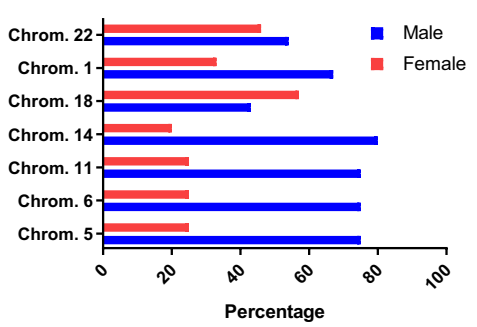

Fig. 2 Summary of copy-number variations $(\mathrm{CNV})$ in pediatric meningiomas derived from methylation analysis. a Representative copynumber plot. b Summary of CNV among all 37 pediatric meningi- omas. Frequency of the most common $\mathrm{CNV}$ (c), and distribution of CNV by WHO grade (d) and sex (e) 

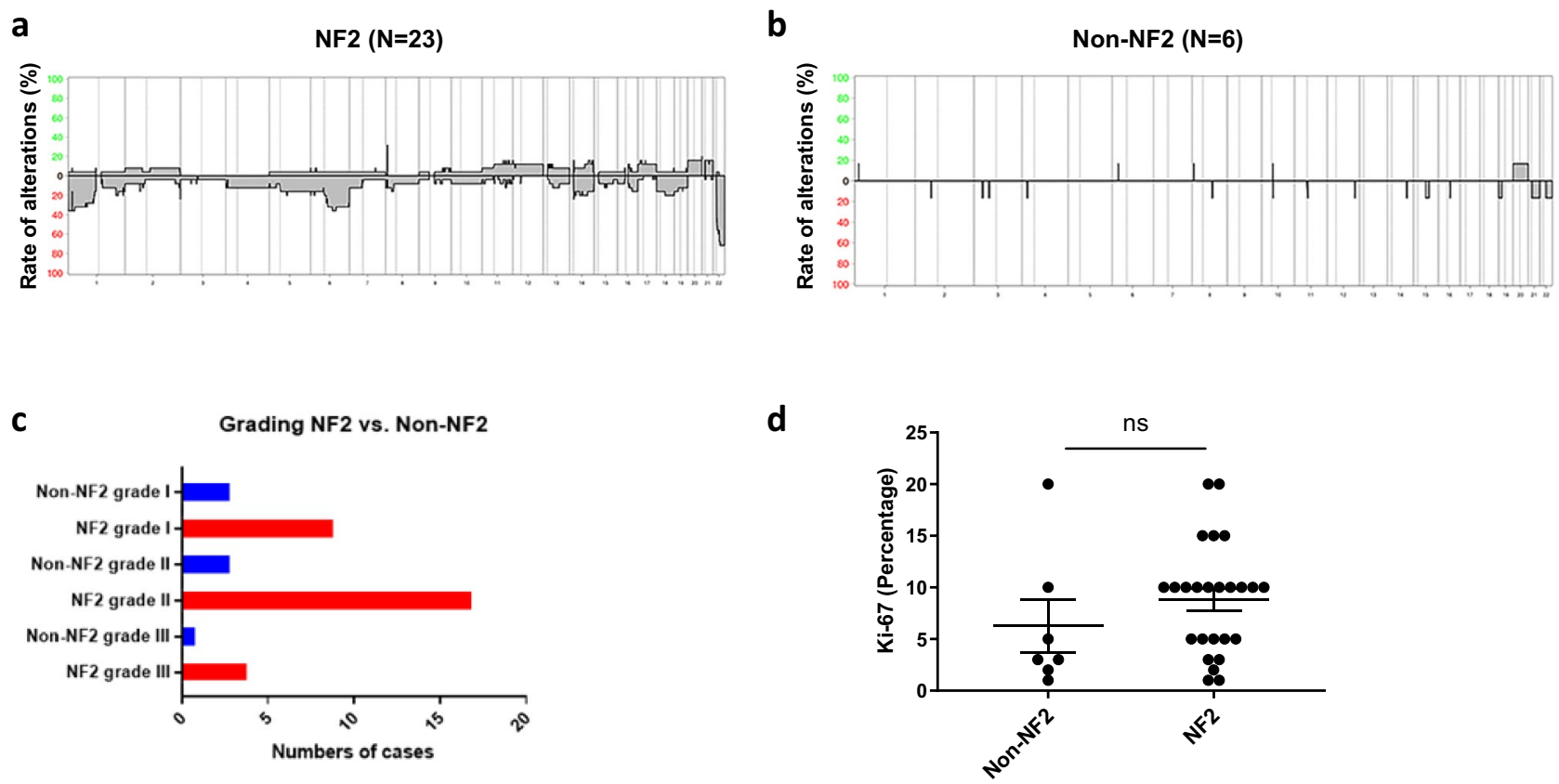

Fig. 3 Comparison of pediatric meningiomas with (NF2) or without (non-NF2) allelic losses at chromosome 22. Summary of CNV in cases with (a) or without (b) NF2-LOH. c Distribution among WHO grades. d Proliferation activity (Ki-67 labeling)

\section{Pediatric meningiomas form three distinct methylation groups}

Next we wondered whether separate methylation groups are present among pediatric meningiomas. Applying a methylation profile-based brain tumor classifier [6], 35/37 (95\%) samples were classified as meningioma. However, one case was classified as high-grade glioma but showed histologically features of a papillary meningioma. Another case, meningioma by histology as well, was classified as a desmoplastic infantile ganglioglioma [DIGG]. Interestingly, a TSNE plot (Figure S6) revealed that the tumor classified as DIGG grouped with other meningiomas (blue arrow), while the papillary meningioma indeed fell into the high-grade glioma group (green arrow).

Unsupervised hierarchical clustering of all 37 pediatric meningiomas revealed separation into three subgroups (Fig. 4a). On the far left, a group of six patients could be distinguished (group 1). Among the remaining 31 cases, two major subgroups were formed, consisting of 15 (group 2A) and 16 (group 2B) cases. Group 1 was comprised of the single papillary meningioma within the series (arrow) and five clear-cell meningiomas. Group 2A with 15 cases consisted mainly of atypical meningiomas $(N=8)$, one clear-cell meningioma, one anaplastic, as well as $5 \mathrm{WHO}$ grade I tumors of meningothelial, fibroblastic, transitional, and psammomatous variant. Group 2B comprising 16 tumors covered 6 WHO grade I tumors, three rhabdoid meningiomas, one chordoid meningioma, and six atypical meningiomas (Fig. 4b). The recurrent tumors which had been undergone WGS grouped into group 2A.

The methylation profile-based separation into three subgroups prompted further comparisons between the proposed methylation subgroups 1,2A and 2B. Loss of chromosome 22 was significantly less frequent in group $2 B$ (Fig. 4c). If the groups were compared regarding the clinical information about signs of NF2, no patient from group 2B had been diagnosed clinically for this disease (Fig. 4d). These findings suggest that group 1 and $2 \mathrm{~A}$ but not group $2 \mathrm{~B}$ are driven by NF2 alterations. Additionally, group 2B showed frequent losses of chromosome 11 (Fig. 4e), while group 1 was characterized by loss of chromosome 19. The proliferation activity, however, was not significantly different between the three groups (supplementary Figure S1).

\section{Panel sequencing}

Because we initially did not detect characteristic somatic mutations known from adult meningiomas by Sanger sequencing, our cohort was further screened for mutations in 130 genes by panel sequencing as previously reported [30]. The oncoplot in Fig. 5 shows the results from 34 cases with sufficient material for analysis, together with clinicopathological data. As already suggested by the methylation profiling and respective copy-number data, the most frequent alteration affected the $N F 2$ gene $(8 / 34,24 \%)$. Additional 
a

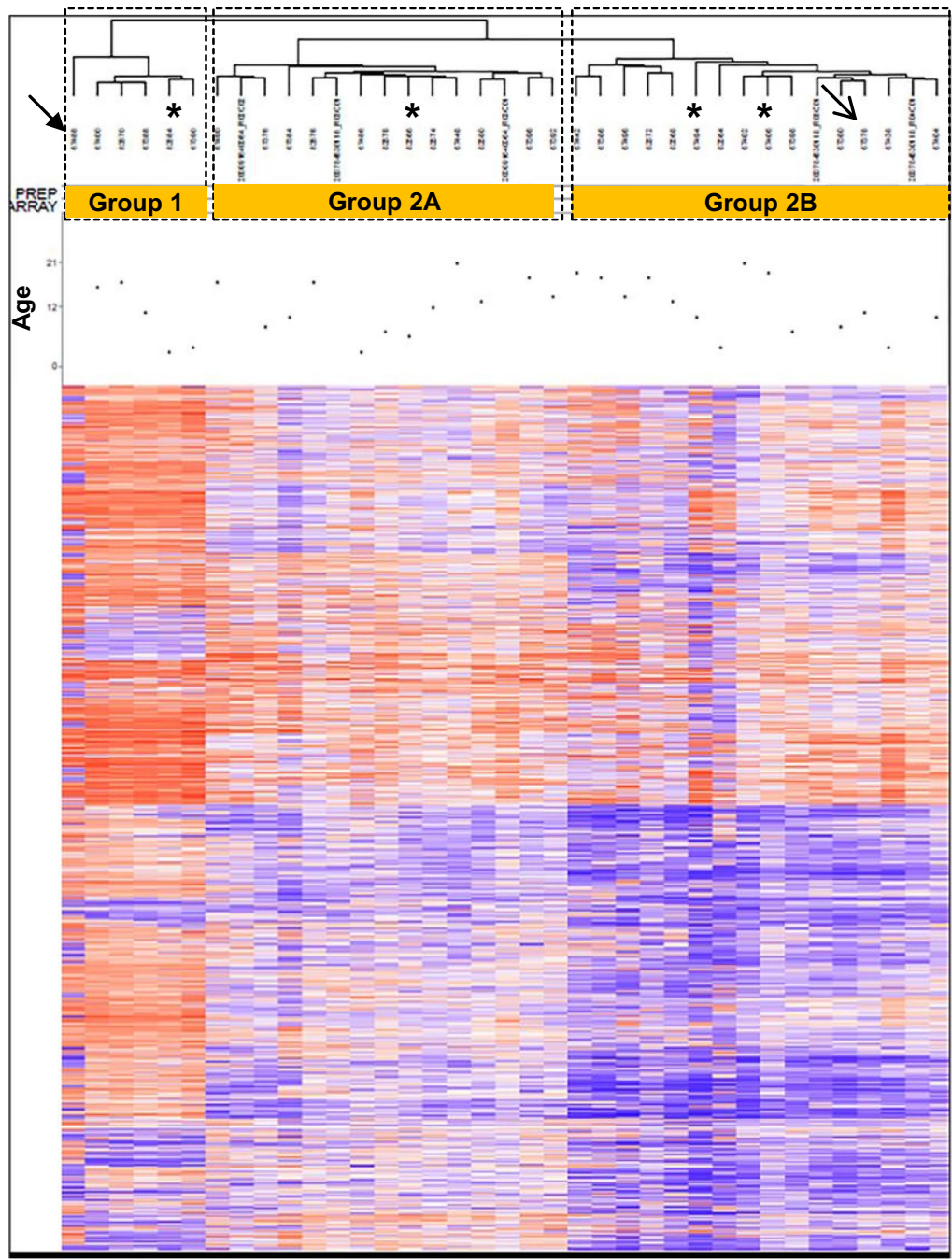

b

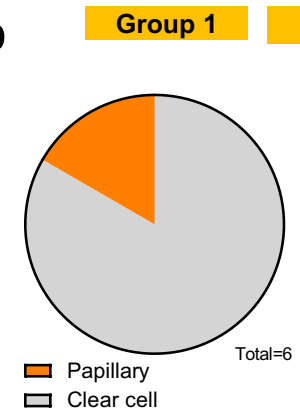

Group 2A

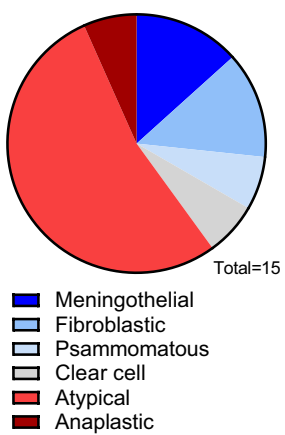

Group 2B

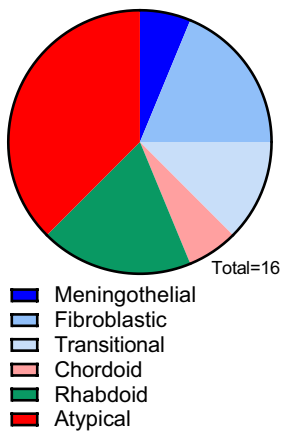

C

Loss of 22 by methylation groups

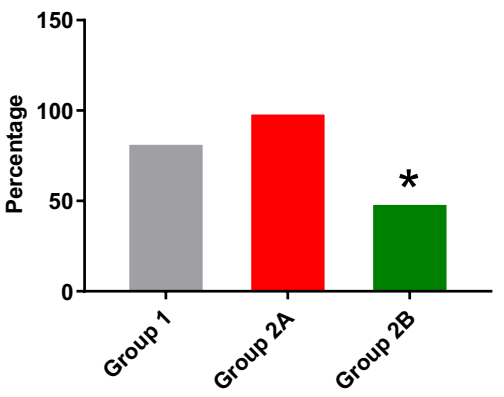

d

Clinical diagnosis of NF2

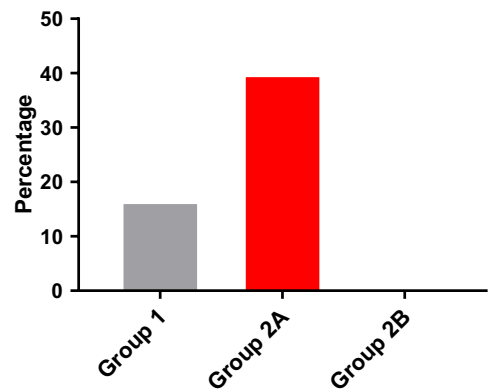

e

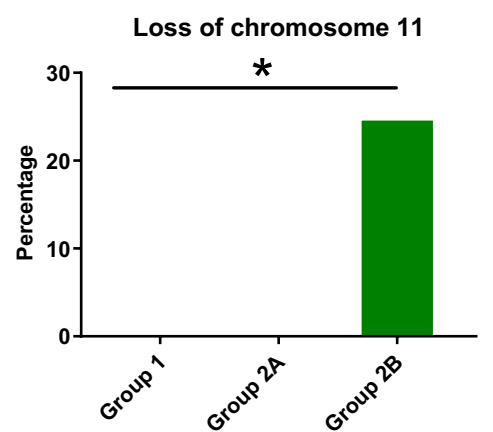

Fig. 4 Methylation profile of 37 pediatric meningiomas. a Unsupervised hierarchical clustering reveals three subgroups (1, 2A, 2B). Arrows indicate tumors not classified as meningioma in the meningioma classifier for adult tumors. Radiation-induced pediatric meningiomas are marked by $(*)$. b Relation between histological variant and methylation subgroup. Frequency of $N F 2-\mathrm{LOH}$ (c) and clinical diagnosis of NF2 (d) among the three subgroups. e Frequency of LOH at chromosome 11 among the three subgroups. Note that c-e display data for 35 cases after exclusion of two cases (arrows) which were not classified as meningioma using the meningioma classifier [31] 


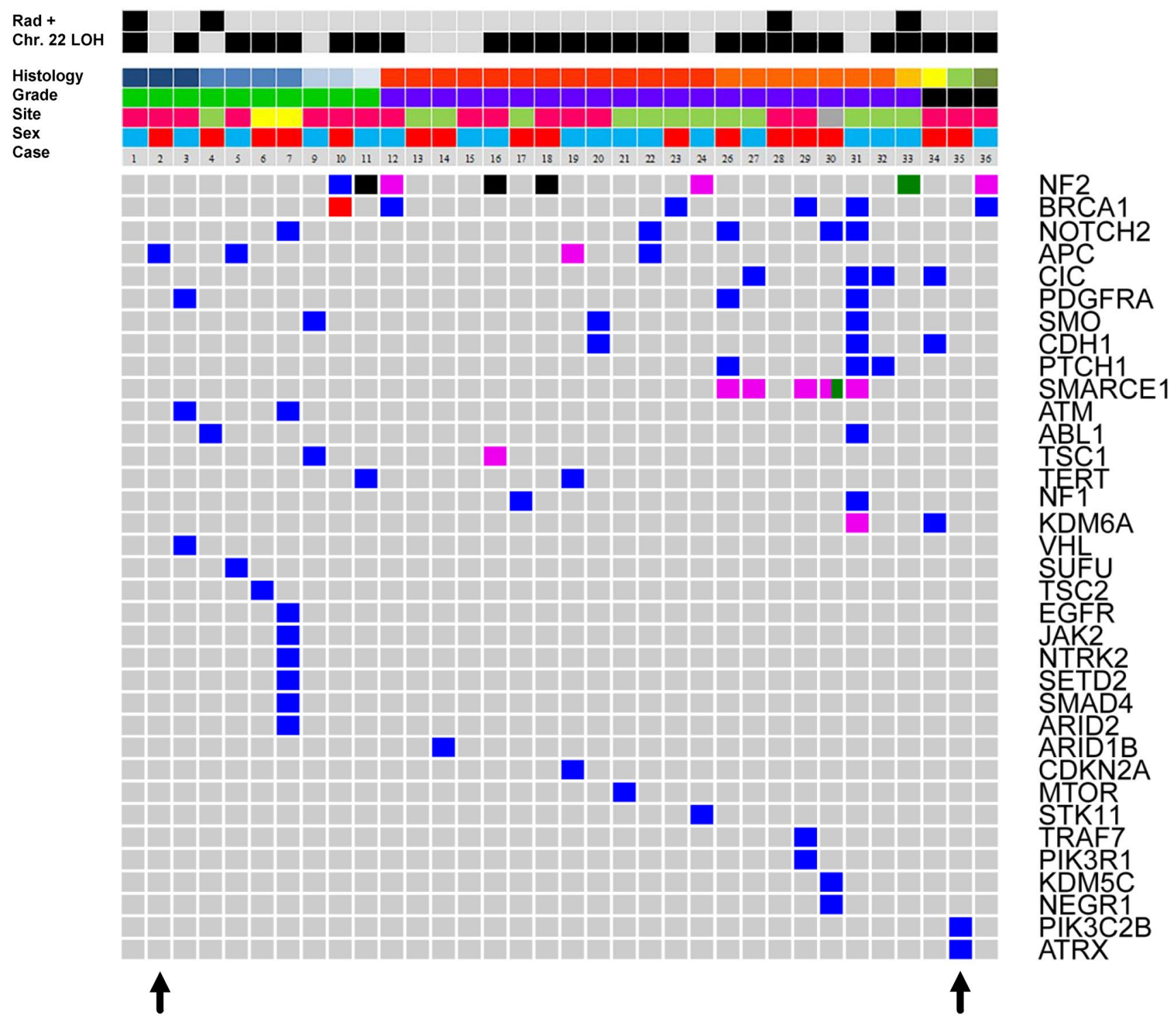

\section{Histological variant WHO grade}

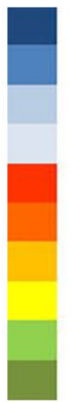

\begin{tabular}{|c|c|c|}
\hline Meningothelial & & WHO grade I \\
\hline Fibroblastic & & WHO grade II \\
\hline Transitional & & WHO grade III \\
\hline Psammomatous & & \\
\hline Atypical & & \\
\hline Clear cell & Sex & \\
\hline Chordoid & & \\
\hline Anaplastic & & Male \\
\hline Papillary & & Female \\
\hline
\end{tabular}

Fig. 5 Oncoplot summarizing relation between histological variant, WHO grade, sex, tumor localization (site), prior irradiation, and mutational spectrum derived from panel sequencing of 130 genes. 34
Localization

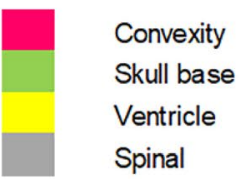

cases were analyzed; for three cases no sufficient material was available. Arrows indicate the two samples from Fig. 4a which were not classified as meningioma

\section{Type of alteration}

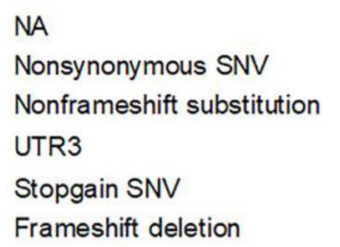

Nonframeshift substitution

Stopgain SNV

Frameshift deletion 
alterations were occasionally found in $B R C A 1, R G P D 3$, $A P C, T S C 1$, and KDM6A with uncertain relevance and unknown germline status. All SMARCE1 alterations were present in clear-cell meningiomas (mutations in 5/7 tumors detected). No hotspot mutations found in adult meningiomas were detected for $S M O, K L F 4$, and $A K T 1$. Moreover, no TERT promoter mutations were detected. Additional immunohistochemistry for BAP1 in two of the three rhabdoid meningiomas showed retained immunoexpression, suggesting absence of BAP1 mutations (Figure S5).

\section{Methylation profiles differ between adult and pediatric meningiomas}

Next, we analyzed the methylation profiles from all 37 pediatric meningiomas together with a group of 105 adult meningiomas which has been described in detail before [31]. As shown in Fig. 6, the vast majority of pediatric meningiomas $(N=28)$ again clustered into a separate group. Moreover, the three subgroups $(1,2 \mathrm{~A}, 2 \mathrm{~B})$ defined above could be distinguished again. The few pediatric tumors clustering with adult malignant (light green) or benign NF2-mutated tumors (light blue) were an anaplastic meningioma, an atypical meningioma, and a fibroblastic meningioma.

\section{Signaling pathways activated in pediatric meningiomas}

To evaluate potential treatment targets in our cohort, we performed immunohistochemical analyses for several proteins involved in essential cellular signaling pathways. A TMA containing 20 pediatric meningiomas was studied by
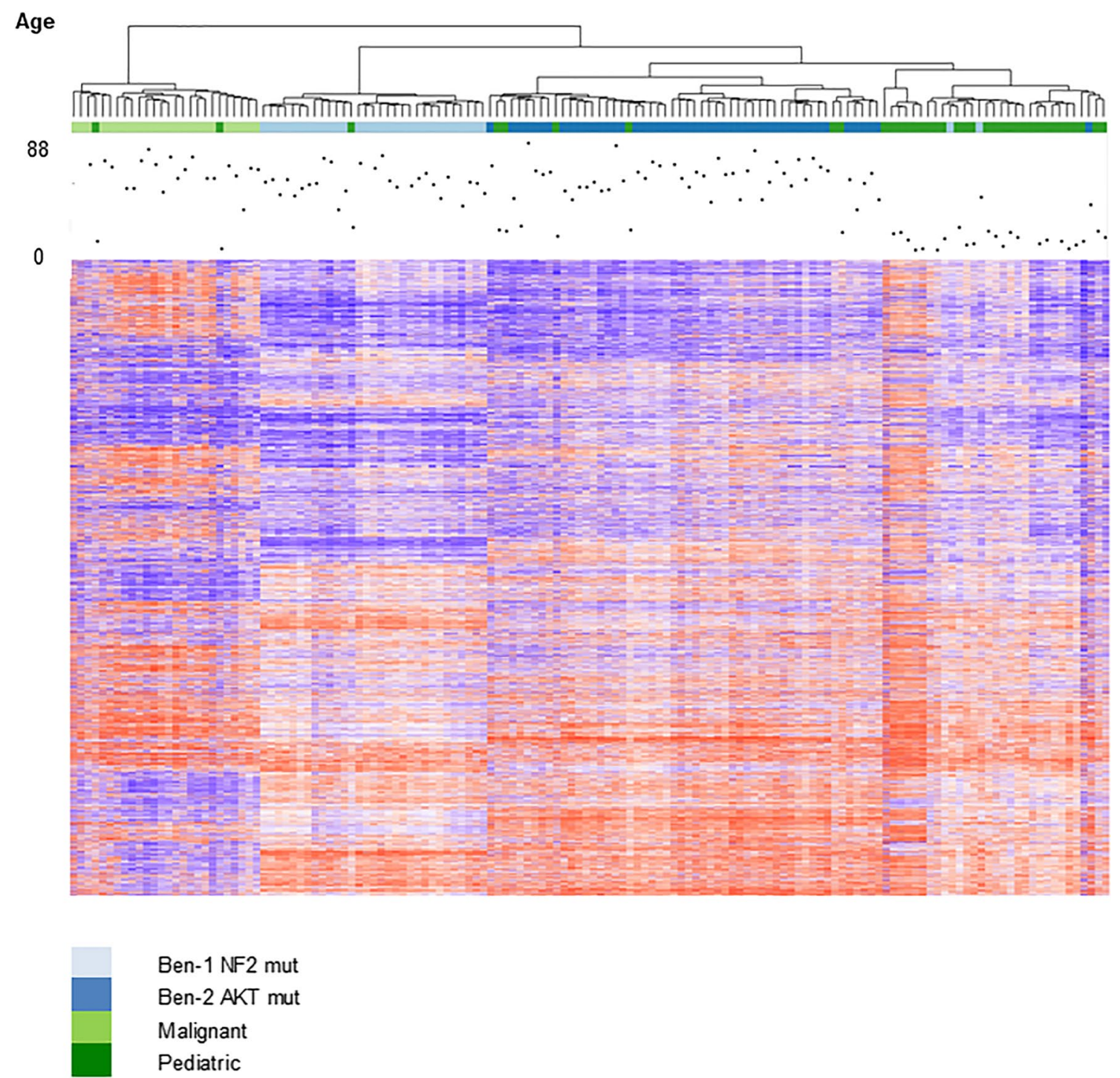

Fig. 6 Unsupervised hierarchical clustering of 105 adult and 37 pediatric meningiomas. Ben-1 and Ben-2 refers to methylation subgroups of adult meningiomas proposed by Sahm et al. [31] 
immunohistochemistry. While for most of the proteins the presence or absence of expression was unrelated to clinical parameters or detected molecular alterations, we found that the expression of p38MAPK was significantly associated with increased mitotic activity $(p=0.036)$ and increased Ki67 proliferation index $(p=0.041)$ (Fig. 7a-c).

\section{Discussion}

We have performed comprehensive molecular analysis of a large group of pediatric meningiomas, revealing that these tumors are substantially different from their adult counterparts by means of histological subtype distribution, methylation profiles, and mutational landscape.

While meningiomas are the most frequent primary intracranial tumors in adults, they are exceptionally rare among children [16] and adolescents [17]. It had been already reported that the gender distribution is different in pediatric meningiomas, with more male patients affected, and that the spectrum of histological variants is different [21]. These epidemiological data were confirmed in our series. A meta-analysis compiling data from 677 pediatric and adolescent meningioma patients showed that patients with NF2 had worse recurrence-free survival (RFS) time than patients without [12]. They also found reduced RFS in patients with grade III meningiomas, compared to grade I/II tumors. However, we did not see a clear reduction of the time to tumor recurrence in grade III meningiomas, although the number of cases with follow-up data available was rather small. In contrast, grade III meningiomas showed similar time to tumor recurrence as grade I meningiomas. This might be explained by the fact that the group of grade III tumors beside anaplastic meningiomas included rare meningioma variants like papillary or rhabdoid meningiomas, for which follow-up data from larger series of pediatric meningiomas are not available.

One of the major findings is the high frequency of chromosome 22 alterations in our cohort. Alterations in the tumor suppressor gene NF2 located on chromosome $22 \mathrm{q}$ are the most common finding in meningiomas occuring in adults. Loss of heterozygosity $(\mathrm{LOH})$ and inactivating NF2 mutations are found in 40-80\%, supporting a classical two-hit hypothesis for meningioma development [27, 28]. The alterations include deletions, insertions, and mutations affecting splice sites, resulting in a non-functional protein [13]. For pediatric meningiomas, Perry et al. have reported a detailed analysis on $N F 2$ in this patient group, with $N F 2$ deletions in $86 \%$ of NF2 patients and $70 \%$ in non-NF2 patients [21]. We found $\mathrm{LOH}$ on chromosome 22 in $76 \%$ of samples, confirming the dominating role of this tumor suppressor gene in pediatric meningiomas.

Recently, a number of Non-NF2 alterations have been identified in sporadic adult meningiomas (reviewed in [23]). The most frequent one affects the TRAF7 (TNF receptor associated factor) gene [7]. Less frequently, alterations in SMO, KLF4, AKT1, PIK3CA are detectable. In our initial Sanger sequencing approach we did not detect any of these alterations, which is in line with recent data from Battu et al. [3]. Recently, Toland et al. reported similar findings with absence of the non-NF2 alterations in pediatric meningiomas [39]. Together with the high frequency of NF2 alterations it can be concluded that pediatric meningiomas are mainly driven by loss of this tumor suppressor.

TERT promoter mutations have been found in about $6 \%$ of adult meningiomas and were associated with higher meningioma grade and early recurrence [30, 37]. Surprisingly, despite the high frequency of aggressive meningioma subtypes, especially atypical meningiomas, no TERT promoter mutations were detected. a

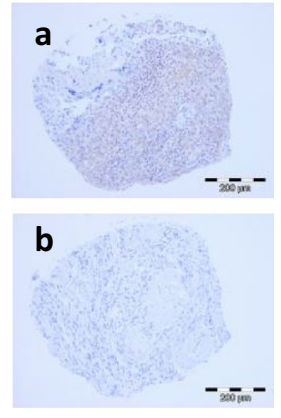

b

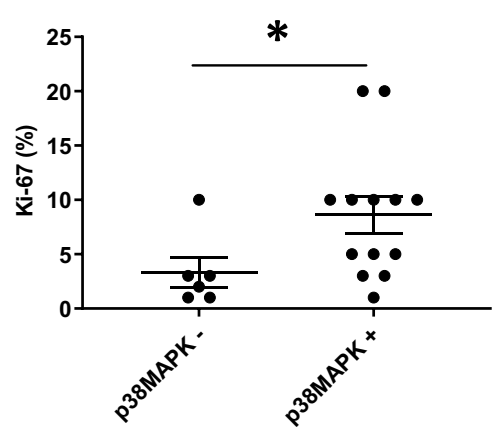

C

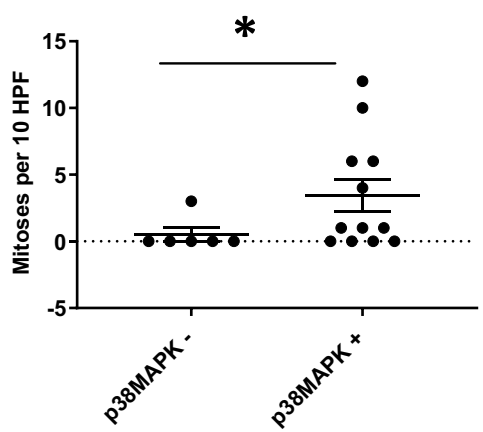

Fig. 7 Analysis of p38MAPK signaling in pediatric meningiomas. Examples of p38MAPK-immunopositive (a) and immunonegative (b) tumor samples derived from a tissue micro array (TMA). Pediatric meningiomas with activation of p38MAPK have higher proliferation activity (b) and mitotic count (c) compared to meningiomas without p38MAPK expression 
The most intriguing finding of our study was the observation that unsupervised clustering of the methylation profile revealed three novel subgroups. Analysis of DNA methylation to characterize brain tumors has been recently introduced in molecular neuropathology as a valuable tool not only to support conventional histological diagnoses, but also to uncover biological relations between morphologically unrelated tumors [6]. For adult meningiomas, Sahm et al. have recently established a methylation-based classification system, separating six different methylation classes with distinct molecular and clinical characteristics [1]. The current study expands this methylation-based classification to pediatric meningiomas, suggesting three distinct subgroups with separate molecular characteristics.

One group (designated group 1) covering 6 pediatric meningiomas was almost exclusively built by clear-cell meningiomas. Clear cell meningiomas are known to be most prevalent in pediatric and adolescent meningiomas [24], and their molecular basis is defined by alterations of the SMARCE1 tumor suppressor gene [8,36]. Consistently, panel sequencing confirmed the presence of SMARCE1 mutations in five of these tumors. In line with previous data, group 1 was additionally characterized by allelic losses at chromosome 22. Another intriguing molecular feature of group 1 was the high frequency of allelic losses at chromosome 19. This aberration has been rarely described in meningiomas in association with high-grade meningiomas [2, 15].

The remaining 31 pediatric meningiomas formed a large group which could be separated into two distinct subgroups designated as group 2A and 2B. Group 2A was dominated by atypical meningiomas WHO grade II. Interestingly, in this group $100 \%$ of cases showed chromosome 22 loss, as well as the highest frequency of clinically diagnosed NF2. Thus, this group can be described as the NF2-driven subgroup of pediatric meningiomas.

The third group, designated group $2 \mathrm{~B}$, was characterized by a mixture of histological variants but included all rhabdoid meningiomas WHO grade III. Moreover, only in this group allelic loss of chromosome 11 were observed, while loss of chromosome 22 was rare and clinical signs of NF2 were absent. Meningiomas with rhabdoid features were initially thought to be characterized by aggressive biology [22] Recently it could be shown that the clinical course of rhabdoid meningiomas largely depends on the loss of the tumor suppressor $B A P 1$, irrespective of the underlying rhabdoid phenotype $[33,34,40]$.

YAP1 fusions have been recently reported among pediatric meningiomas [35]. However, chromosomal rearrangement around the $Y A P I$ locus serving as a surrogate for $Y A P 1$ alterations were not detected in our cohort, while RNA sequening would be required to address this question appropriately.
Another striking observation is that the methylation profile of pediatric meningiomas differs clearly from the one observed in adult meningioma patients. By analyzing pediatric and adult tumors together the pediatric group still was clearly separated. Despite the high frequency of NF2 alterations in the pediatric group, the tumors did not fall into the cluster of NF2-altered adult meningiomas. This indicates that the underlying biology is substantially different between pediatric and adult meningiomas.

The lack of NF2 patients being represented in the 2B methylation cluster could have clinical implications. While approximately $40 \%$ of young patients presenting with a solitary meningioma have an underlying germline NF2 mutation, the diagnosis can be difficult to ascertain genetically due to mosaic status and may require years to confirm clinically [19]. Moreover, in our retrospective series it appeared difficult to trace back clinical features according to the consensus criteria [9]. If our findings can be confirmed in a larger cohort of NF2 meningioma patients, methylation profiling could provide a valuable diagnostic adjunct.

Taken together, our data show that pediatric meningiomas can be separated into groups with distinct morphological and molecular features, and that pediatric meningiomas are distinct from adult counterparts despite sharing a high frequency of NF2 alterations. However, compared with adult counterparts and the well-established methylation classes with clear prognostic implications [31], for pediatric meningiomas the establishment of prognostic groups based on the methylation classification appears less likely, given the low frequency of meningiomas in this age group.

Supplementary Information The online version contains supplementary material available at https://doi.org/10.1007/s00401-021-02351-x.

Acknowledgements We thank the DKFZ Heidelberg Center for Personalized Oncology (DKFZ-HIPO) for technical support and funding through HIPO_047. We further thank the DKFZ Omics IT and Data Management Core Facility (ODCF) and DKFZ Genomics and Proteomics Core Facility for technical support. The work was supported by a grant from the German Children Cancer Aid, grant number \#2013.04. We thank Dr. Jeffrey Leonhard, Department of Neurosurgery, Washington University School of Medicine, for providing material. We also thank the Children's Discovery Institute (St. Louis, MO) for support. U.S. was supported by the Fördergemeinschaft Kinderkrebszentrum Hamburg.

Funding Open Access funding enabled and organized by Projekt DEAL.

Open Access This article is licensed under a Creative Commons Attribution 4.0 International License, which permits use, sharing, adaptation, distribution and reproduction in any medium or format, as long as you give appropriate credit to the original author(s) and the source, provide a link to the Creative Commons licence, and indicate if changes were made. The images or other third party material in this article are included in the article's Creative Commons licence, unless indicated otherwise in a credit line to the material. If material is not included in 
the article's Creative Commons licence and your intended use is not permitted by statutory regulation or exceeds the permitted use, you will need to obtain permission directly from the copyright holder. To view a copy of this licence, visit http://creativecommons.org/licenses/by/4.0/.

\section{References}

1. Agnihotri S, Suppiah S, Tonge PD, Jalali S, Danesh A, Bruce JP et al (2017) Therapeutic radiation for childhood cancer drives structural aberrations of NF2 in meningiomas. Nat Commun 8:186. https://doi.org/10.1038/s41467-017-00174-7

2. Basaran R, Uslu S, Gucluer B, Onoz M, Isik N, Tiryaki M et al (2016) Impact of $1 p / 19 q$ codeletion on the diagnosis and prognosis of different grades of meningioma. Br J Neurosurg 30:571576. https://doi.org/10.1080/02688697.2016.1181155

3. Battu S, Kumar A, Pathak P, Purkait S, Dhawan L, Sharma MC et al (2018) Clinicopathological and molecular characteristics of pediatric meningiomas. Neuropathology 38:22-33. https://doi.org/ 10.1111/neup. 12426

4. Boetto J, Bielle F, Sanson M, Peyre M, Kalamarides M (2017) SMO mutation status defines a distinct and frequent molecular subgroup in olfactory groove meningiomas. Neuro Oncol 19:345351. https://doi.org/10.1093/neuonc/now276

5. Brastianos PK, Horowitz PM, Santagata S, Jones RT, McKenna A, Getz $\mathrm{G}$ et al (2013) Genomic sequencing of meningiomas identifies oncogenic SMO and AKT1 mutations. Nat Genet 45:285-289

6. Capper D, Jones DTW, Sill M, Hovestadt V, Schrimpf D, Sturm D et al (2018) DNA methylation-based classification of central nervous system tumours. Nature 555:469-474. https://doi.org/10. 1038/nature26000

7. Clark VE, Erson-Omay EZ, Serin A, Yin J, Cotney J, Ozduman K et al (2013) Genomic analysis of non-NF2 meningiomas reveals mutations in TRAF7, KLF4, AKT1, and SMO. Science 339:1077-1080

8. Evans LT, Hoff JV, Hickey WF, Smith MJ, Evans DG, Newman WG et al (2015) SMARCE1 mutations in pediatric clear cell meningioma: case report. J Neurosurg Pediatr 16:296-300. https://doi. org/10.3171/2015.3.PEDS14417

9. Halliday D, Parry A, Evans DG (2019) Neurofibromatosis type 2 and related disorders. Curr Opin Oncol 31:562-567. https://doi. org/10.1097/cco.0000000000000579

10. Jiang Y, Qiu Y, Minn AJ, Zhang NR (2016) Assessing intratumor heterogeneity and tracking longitudinal and spatial clonal evolutionary history by next-generation sequencing. Proc Natl Acad Sci USA 113:E5528-5537. https://doi.org/10.1073/pnas.1522203113

11. Jones DT, Hutter B, Jager N, Korshunov A, Kool M, Warnatz HJ et al (2013) Recurrent somatic alterations of FGFR1 and NTRK2 in pilocytic astrocytoma. Nat Genet 45:927-932. https://doi.org/ 10.1038/ng.2682

12. Kotecha RS, Pascoe EM, Rushing EJ, Rorke-Adams LB, Zwerdling T, Gao X et al (2011) Meningiomas in children and adolescents: a meta-analysis of individual patient data. Lancet Oncol 12:1229 1239. https://doi.org/10.1016/s1470-2045(11)70275-3

13. Lekanne Deprez RH, Bianchi AB, Groen NA, Seizinger BR, Hagemeijer A, Drunen E et al (1994) Frequent NF2 gene transcript mutations in sporadic meningiomas and vestibular schwannomas. Am J Hum Genet 54:1022-1029

14. Louis DN, Perry A, Reifenberger G, von Deimling A, FigarellaBranger D, Cavenee WK et al (2016) The 2016 World Health Organization classification of tumors of the central nervous system: a summary. Acta Neuropathol. https://doi.org/10.1007/ s00401-016-1545-1
15. Nagasaka T, Gunji M, Hosokai N, Hayashi K, Fujino M, Ikeda $\mathrm{H}$ et al (2010) Fluorescent in situ hybridization 1p/19q deletion/ imbalance analysis of low-grade and atypical meningiomas. Neurol Med Chir (Tokyo) 50:27-32. https://doi.org/10.2176/nmc.50. 27 (discussion 32)

16. Ostrom QT, de Blank PM, Kruchko C, Petersen CM, Liao P, Finlay JL et al (2015) Alex's Lemonade Stand Foundation Infant and childhood primary brain and central nervous system tumors diagnosed in the United States in 2007-2011. Neuro Oncol 16:x1-x36. https://doi.org/10.1093/neuonc/nou327

17. Ostrom QT, Gittleman H, de Blank PM, Finlay JL, Gurney JG, McKean-Cowdin R et al (2016) American brain tumor association adolescent and young adult primary brain and central nervous system tumors diagnosed in the United States in 2008-2012. Neuro Oncol 18:i1-i50. https://doi.org/10.1093/neuonc/nov297

18. Pachow D, Andrae N, Kliese N, Angenstein F, Stork O, WilischNeumann A et al (2013) mTORC1 inhibitors suppress meningioma growth in mouse models. Clin Cancer Res 19:1180-1189

19. Pathmanaban ON, Sadler KV, Kamaly-Asl ID et al (2017) Association of genetic predisposition with solitary schwannoma or meningioma in children and young adults. JAMA Neurol 74:11231129. https://doi.org/10.1001/jamaneurol.2017.1406

20. Perry A, Dehner LP (2003) Meningeal tumors of childhood and infancy. An update and literature review. Brain Pathol (Zurich) 13:386-408

21. Perry A, Giannini C, Raghavan R, Scheithauer BW, Banerjee R, Margraf L et al (2001) Aggressive phenotypic and genotypic features in pediatric and NF2-associated meningiomas: a clinicopathologic study of 53 cases. J Neuropathol Exp Neurol 60:994-1003

22. Perry A, Scheithauer BW, Stafford SL, Abell-Aleff PC, Meyer FB (1998) "Rhabdoid" meningioma: an aggressive variant. Am J Surg Pathol 22:1482-1490

23. Preusser M, Brastianos PK, Mawrin C (2018) Advances in meningioma genetics: novel therapeutic opportunities. Nat Rev Neurol 14:106-115. https://doi.org/10.1038/nrneurol.2017.168

24. Raffalli-Ebezant H, Rutherford S, Stivaros S, Kelsey A, Smith M, Evans D et al (2015) Pediatric intracranial clear cell meningioma associated with a germline mutation of SMARCE1: a novel case. Childs Nerv Syst 31:441-447. https://doi.org/10. 1007/s00381-014-2558-5

25. Reuss DE, Piro RM, Jones DT, Simon M, Ketter R, Kool M et al (2013) Secretory meningiomas are defined by combined KLF4 K409Q and TRAF7 mutations. Acta Neuropathol 125:351-358

26. Rochat P, Johannesen HH, Gjerris F (2004) Long-term follow up of children with meningiomas in Denmark: 1935 to 1984. J Neurosurg 100:179-182. https://doi.org/10.3171/ped.2004. 100.2.0179

27. Ruttledge MH, Sarrazin J, Rangaratnam S, Phelan CM, Twist $\mathrm{E}$, Merel P et al (1994) Evidence for the complete inactivation of the NF2 gene in the majority of sporadic meningiomas. Nat Genet 6:180-184

28. Ruttledge MH, Xie YG, Han FY, Peyrard M, Collins VP, Nordenskjold M et al (1994) Deletions on chromosome 22 in sporadic meningioma. Genes Chromosom Cancer 10:122-130

29. Sahm F, Schrimpf D, Jones DT, Meyer J, Kratz A, Reuss D et al (2016) Next-generation sequencing in routine brain tumor diagnostics enables an integrated diagnosis and identifies actionable targets. Acta Neuropathol 131:903-910. https://doi.org/10.1007/ s00401-015-1519-8

30. Sahm F, Schrimpf D, Olar A, Koelsche C, Reuss D, Bissel J et al (2016) TERT promoter mutations and risk of recurrence in meningioma. J Natl Cancer Inst. https://doi.org/10.1093/jnci/ djv377

31. Sahm F, Schrimpf D, Stichel D, Jones DT, Hielscher T, Schefzyk $S$ et al (2017) DNA methylation-based classification and grading 
system for meningioma: a multicentre, retrospective analysis. Lancet Oncol. https://doi.org/10.1016/s1470-2045(17)30155-9

32. Sahm F, Toprak UH, Hubschmann D, Kleinheinz K, Buchhalter I, Sill M et al (2017) Meningiomas induced by low-dose radiation carry structural variants of NF2 and a distinct mutational signature. Acta Neuropathol 134:155-158. https://doi.org/10.1007/ s00401-017-1715-9

33. Shankar GM, Abedalthagafi M, Vaubel RA, Merrill PH, Nayyar N, Gill CM et al (2017) Germline and somatic BAP1 mutations in high-grade rhabdoid meningiomas. Neuro Oncol 19:535-545. https://doi.org/10.1093/neuonc/now235

34. Shankar GM, Santagata S (2017) BAP1 mutations in high-grade meningioma: implications for patient care. Neuro Oncol 19:14471456. https://doi.org/10.1093/neuonc/nox094

35. Sievers P, Chiang J, Schrimpf D, Stichel D, Paramasivam N, Sill $M$ et al (2020) YAP1-fusions in pediatric NF2-wildtype meningioma. Acta Neuropathol 139:215-218. https://doi.org/10.1007/ s00401-019-02095-9

36. Smith MJ, Ahn S, Lee JI, Bulman M, Plessis DD, Suh YL (2017) SMARCE1 mutation screening in classification of clear cell meningiomas. Histopathology 70:814-820. https://doi.org/10.1111/ his. 13135

37. Spiegl-Kreinecker S, Lötsch D, Neumayer K, Kastler L, Gojo J, Pirker $\mathrm{C}$ et al (2018) TERT promoter mutations are associated with poor prognosis and cell immortalization in meningioma. Neuro Oncol 20:1584-1593. https://doi.org/10.1093/neuonc/ noy 104

38. Sturm D, Witt H, Hovestadt V, Khuong-Quang DA, Jones DT, Konermann C et al (2012) Hotspot mutations in H3F3A and IDH1 define distinct epigenetic and biological subgroups of glioblastoma. Cancer Cell 22:425-437. https://doi.org/10.1016/j.ccr.2012.08.024

39. Toland A, McNulty SN, Pekmezci M, Evenson M, Huntoon K, Pierson CR et al (2020) Pediatric meningioma: a clinicopathologic and molecular study with potential grading implications. Brain Pathol (Zurich) 30:1134-1143. https://doi.org/10.1111/bpa.12884

40. Vaubel RA, Chen SG, Raleigh DR, Link MJ, Chicoine MR, Barani I et al (2016) Meningiomas with rhabdoid features lacking other histologic features of malignancy: a study of 44 cases and review of the literature. J Neuropathol Exp Neurol 75:44-52. https://doi. org/10.1093/jnen/nlv006

41. von Spreckelsen N, Waldt N, Poetschke R, Kesseler C, Dohmen H, Jiao HK et al (2020) KLF4(K409Q)-mutated meningiomas show enhanced hypoxia signaling and respond to mTORC1 inhibitor treatment. Acta Neuropathol Commun 8:41. https://doi.org/10. 1186/s40478-020-00912-x

42. Yesiloz U, Kirches E, Hartmann C, Scholz J, Kropf S, Sahm F et al (2017) Frequent AKT1E17K mutations in skull base meningiomas are associated with mTOR and ERK1/2 activation and reduced time to tumor recurrence. Neuro Oncol 19(8):1088-1096. https://doi.org/10.1093/neuonc/nox018

Publisher's Note Springer Nature remains neutral with regard to jurisdictional claims in published maps and institutional affiliations.

\section{Authors and Affiliations}

\section{Elmar Kirches ${ }^{1} \cdot$ Felix Sahm $^{3} \cdot$ Andrey Korshunov $^{3} \cdot$ Christina Bluecher $^{1} \cdot$ Natalie Waldt $^{1} \cdot$ Siegfried Kropf $^{2}$. Daniel Schrimpf ${ }^{3}$. Philipp Sievers ${ }^{3}$. Damian Stichel ${ }^{3}$. Ulrich Schüller ${ }^{4,5}$. Jens Schittenhelm ${ }^{6}$. Markus J. Riemenschneider ${ }^{7}$. Matthias A. Karajannis ${ }^{15,18}$. Arie Perry $^{16} \cdot$ Torsten Pietsch $^{8}$. Svenja Boekhoff ${ }^{9}$. David Capper ${ }^{10,11} \cdot$ Katja Beck $^{12}$. Nagarajan Paramasivam ${ }^{12}$. Matthias Schlesner ${ }^{12,20}$. Priscilla K. Brastianos ${ }^{17}$. Hermann L. Müller ${ }^{9}$. Stefan M. Pfister ${ }^{13,14}$. Christian Mawrin ${ }^{1,19}$}

1 Department of Neuropathology, Otto-von-Guericke University Magdeburg, Magdeburg, Germany

2 Biometry and Medical Informatics, Otto-von-Guericke University Magdeburg, Magdeburg, Germany

3 Department of Neuropathology, University Hospital Heidelberg, Heidelberg, Germany

4 Department of Neuropathology, University Hospital Hamburg, Hamburg, Germany

5 Department of Pediatric Hematology and Oncology, Research Institute Children's Cancer Center Hamburg, University Hospital Hamburg, Hamburg, Germany

6 Department of Neuropathology, University Hospital Tuebingen, Tuebingen, Germany

7 Department of Neuropathology, University Hospital Regensburg, Regensburg, Germany

8 Department of Neuropathology, University Hospital Bonn, Bonn, Germany

9 Department of Pediatric Oncology, University Children's Hospital, Oldenburg, Germany
10 Department of Neuropathology, Berlin Institute of Health, Charité-Universitätsmedizin Berlin, Freie Universität Berlin, Humboldt-Universität Zu Berlin, 10117 Berlin, Germany

11 German Cancer Consortium (DKTK), German Cancer Research Center (DKFZ), Partner Site Berlin, Heidelberg, Germany

12 Bioinformatics and Omics Data Analytics (B240), German Cancer Research Center (DKFZ), Heidelberg, Germany

13 KiTZ, DKFZ and DKTK, Hopp Children's Cancer Center Heidelberg, Heidelberg, Germany

14 Division of Pediatric Neurooncology, Department of Pediatric Hematology and Oncology, Heidelberg University Hospital, Heidelberg, Germany

15 Department of Pediatric Hematology/Oncology, NYU Langone Medical Center, New York, NY, USA

16 University of California, San Francisco, USA

17 Massachusetts General Hospital, Boston, USA

18 Present Address: Pediatric Neuro-Oncology Service, Department of Pediatrics, Memorial Sloan Kettering Cancer Center, New York, NY, USA 
19 Center for Behavioral Brain Sciences (CBBS), Magdeburg, Germany

20 Present Address: Biomedical Informatics, Data Mining and Data Analytics, Faculty of Applied Computer Science and Medical Faculty, University of Augsburg, Augsburg, Germany 